\title{
Quelle est la motivation des étudiants en médecine pour l'apprentissage de la lecture critique d'article?
}

\section{What motivates medical students to learn critical reading of scientific articles?}

\author{
Jérémie JEGU ${ }^{1,2}$, Marc BRAUN ${ }^{3}$, Thierry PELACCIA ${ }^{4}$
}

1 Laboratoire d'Épidémiologie et de Santé Publique, EA3430, FMTS, Université de Strasbourg, 4 rue Kirschleger, 67085 Strasbourg Cedex, France

2 Service de santé publique, Hôpitaux Universitaires de Strasbourg, 1 place de l’hôpital, 67091 Strasbourg Cedex, France

3 Département de pédagogie et CUESIM, Faculté de médecine, Université de Lorraine à Nancy, 9 avenue de la Forêt de Haye, 54500 Vandoeuvre-lès-Nancy

4 Laboratoire de recherche en pédagogie des sciences de la santé, Département de pédagogie médicale, Faculté de médecine de Strasbourg, 4 rue Kirschleger, 67085 Strasbourg Cedex, France

Manuscrit reçu le 7 mars 2014 ; commentaires éditoriaux formulés aux auteurs le 17 septembre 2014 et le 5 octobre 2014 ; accepté pour publication le 6 octobre 2014

\section{Mots-clés \\ Motivation ; Lecture critique d'article ; Médecine basée sur les preuves ; Évaluation des connaissances}

Résumé - Contexte : L'acquisition des connaissances méthodologiques nécessaires à la lecture critique d'articles scientifiques (LCA) est potentiellement peu motivante pour les étudiants en médecine. But : Décrire la motivation des étudiants en médecine pour la LCA et étudier l'impact de celle-ci sur leur performance. Méthodes : Tous les étudiants inscrits en DCEM4 à Strasbourg et à Nancy ont été invités à remplir un auto-questionnaire standardisé en ligne. Cinq composantes motivationnelles ont été explorées : la motivation intrinsèque (MI), la motivation extrinsèque (ME), la perception de la valeur de la tâche (PVT), la perception d'autonomie et de contrôle (PCO) et le sentiment d'efficacité personnelle (SEP). La performance était mesurée par le score (note) obtenu à l'examen de LCA à Strasbourg et par le rang de classement aux épreuves classantes nationales (ECN). Résultats : Au total, 205 questionnaires ont été complétés, soit une participation de 35,4\%. La ME était plus importante que la MI (médiane de 5,00 versus 3,67). La PCO était à un niveau assez élevé (médiane de 4,67), suivie de la PVT (médiane de 4,00) et du SEP (médiane de 3,33). Seul le SEP était corrélé de manière significative au score de LCA à Strasbourg (coefficient de corrélation 0,242, $p=0,018$ ). Cette association n'était plus significative lorsque l'on considérait le rang de classement aux $\operatorname{ECN}(p=$ 0,363). Conclusions : Le SEP était la composante motivationnelle la plus altérée pour la 
LCA. Ceci dégage des perspectives d'actions pédagogiques pour renforcer la qualité des apprentissages réalisés par les étudiants dans le domaine de la LCA.

Keywords

Critical reading; motivation; evidence-based medicine; educational measurement
Abstract - Background: Although learning methodological principles is a prerequisite for effective critical reading of scientific articles (CRA), this may also lead to low motivation among medical students. Objective: Describing the motivation of medical students for CRA and assessing its impact on student performance. Material and methods: All sixth year medical students enrolled in Strasbourg and Nancy (France) were invited to fill out an online standardized self-administered questionnaire. Five motivational components were examined: intrinsic goal orientation (IGO), extrinsic goal orientation (EGO), task value (TV), control of learning beliefs (CLB) and self-efficacy for learning and performance (SELP). Performance was measured using scores in the previous CRA exam in Strasbourg, and the ranking in the French "Épreuves Classantes Nationales" (ECN) (national ranking exams). Results: A total of 205 questionnaires were completed, i.e. a 35.4\% participation rate. The EGO ranked higher than the IGO (median of 5.00 versus 3.67 ). The CLB level was somewhat high (median of 4.67), followed by the TV (median of 4.00) and the SELP (median of 3.33). The SELP was the only component statistically correlated with the CRA exam performance in Strasbourg (correlation coefficient of $0.242, p=0.018$ ). This association was not significant when taking into account the ECN ranking $(p=0.363)$. Findings: The SELP was the most altered motivational component with respect to CRA. It provides opportunities to implement motivating teaching strategies in order to improve ACR learning among medical students.

\section{Contexte et problématique}

Dans le contexte d'un exercice de la médecine basé sur les preuves, les médecins doivent se tenir informés des derniers développements concernant leurs pratiques, notamment grâce à la lecture d'articles scientifiques. Cependant, la lecture de tels articles nécessite certaines compétences en épidémiologie et en biostatistique, qui font souvent défaut chez les internes, comme cela a été récemment souligné dans une étude américaine ${ }^{[1]}$. En effet, la bonne compréhension des études de cohorte, des études cas-témoin ou des études transversales peut nécessiter certaines connaissances spécifiques à l'épidémiologie. Par ailleurs, la lecture d'articles portant sur l'évaluation d'un traitement, d'une procédure diagnostique ou sur l'estimation d'un pronostic peut faire appel à des compétences en méthodologie pour l'interprétation des tests statistiques, la discussion d'éventuels biais et l'appréciation de la généralisabilité des résultats à la pratique clinique.

En France, les étudiants en médecine sont préparés à critiquer de façon autonome leur documentation professionnelle dans le cadre de l'enseignement de la lecture critique d'article (LCA) ${ }^{[2]}$. Les épreuves classantes nationales (ECN), qui ont pour but de classer les étudiants à l'issue du deuxième cycle des études médicales (DCEM) comportent depuis l'année 2009, en plus des dossiers cliniques, une épreuve de LCA $^{[3]}$. Cette épreuve, qui compte pour $10 \%$ du score global depuis 2010, exerce un effet notable sur la performance des étudiants, permettant à certains de gagner ou de perdre de nombreuses places d'une manière comparable aux dossiers cliniques traditionnels ${ }^{[4,5]}$. Les enjeux liés à la formation à la LCA sont donc majeurs en France, et ceci à un double niveau : performance aux ECN d'une part et entretien des compétences dans le cadre de la pratique autonome d'autre part.

Malgré ces enjeux, l'apprentissage de la LCA n'a été proposé que récemment aux étudiants en médecine. De plus, les connaissances méthodologiques nécessaires à la pratique de cet exercice (épidémiologie, biostatistique) peuvent sembler éloignées de celles nécessaires à la pratique clinique, ce qui provoque souvent une réaction de défiance de la part des étudiants, pouvant se traduire par un manque de motivation. 
Or, la motivation, c'est à dire les facteurs internes et/ou externes à l'origine de l'engagement en formation et de l'investissement dans les activités $\mathrm{d}^{\text {'apprentissage }}{ }^{[6]}$ est un déterminant majeur de la qualité des apprentissages et du niveau de performance atteint. Parmi les composantes de la motivation, on distingue habituellement la motivation intrinsèque (moteur de la réalisation d'un acte dont l'individu retire du plaisir ou de la satisfaction) et la motivation extrinsèque (moteur de la réalisation d'un acte en vue de l'obtention d'une récompense ou de l'évitement d'une sanction) ${ }^{[6]}$. À partir de ces deux composantes, Ryan et Deci ont défini le degré d'autodétermination, construit dont la valeur est d'autant plus importante que la motivation intrinsèque est élevée $^{[7]}$. Les travaux issus de la théorie de l'autodétermination ont par ailleurs montré qu'il existe une corrélation entre le degré d'autodétermination et les conséquences positives en terme d'implication, de flexibilité cognitive, de persévérance et de performance dans les activités entreprises, notamment celles liées à l'apprentissage ${ }^{[6]}$.

Dans ce contexte, ce travail de recherche avait pour objectif de décrire la motivation des étudiants en médecine pour l'apprentissage des principes d'épidémiologie et de biostatistique nécessaires à la pratique de la LCA, et d'étudier les associations entre les différentes composantes de la motivation et la performance des étudiants.

\section{Méthodes}

La population étudiée comportait tous les étudiants en quatrième année du deuxième cycle des études médicales (DCEM4) inscrits à la faculté de médecine de Strasbourg et de Nancy pour l'année universitaire 2012-2013. Ces étudiants ont été invités à remplir un questionnaire auto-administré en ligne via un message électronique personnalisé. En cas de nonréponse, deux relances ont été réalisées.

Dans le cadre de ce travail de recherche, nous nous sommes intéressés à la motivation des étudiants pour l'apprentissage des compétences en épidémiologie et biostatistique nécessaires à la LCA. Le niveau de motivation a été mesuré au moyen d'une version adaptée en français du questionnaire «Motivated Strategies for Learning Questionnaire» (MSLQ) développé par Pintrich et al. ${ }^{[8]}$. En plus de la motivation intrinsèque (MI) et de la motivation extrinsèque (ME), trois composantes ont été explorées $^{[6,9]}$ :

- La perception de la valeur de la tâche (PVT), qui traduit le jugement que porte l'apprenant sur l'intérêt à réaliser une activité au regard de ses objectifs et de ses attentes.

- La perception d'autonomie et de contrôle (PCO), qui correspond au degré de contrôle qu'un apprenant croit exercer sur le déroulement d'une activité.

- Le sentiment d'efficacité personnelle (SEP), qui traduit la croyance que possède un individu à l'égard de ses compétences pour atteindre un but.

Comme le questionnaire était auto-administré par voie électronique, nous avons minimisé le risque de réponses incomplètes en proposant aux étudiants une version réduite du questionnaire à 15 items (contre 26 pour la version originale du MSLQ). Le choix des questions s'est fondé sur les résultats de l'étude de validation du MSLQ, en retenant les trois questions qui exploraient le mieux les différentes composantes étudiées ${ }^{[8]}$. Pour chaque question, les étudiants devaient renseigner leur degré d'accord avec les affirmations proposées sur une échelle de Likert graduée de 1 (pas du tout d'accord) à 7 (tout à fait d'accord). Les valeurs des différentes composantes ont été obtenues en calculant la moyenne des questions correspondantes, un score élevé traduisant une valeur élevée pour la composante motivationnelle explorée ${ }^{[8]}$. Les questionnaires ont été complétés en ligne du 08 mai 2013 au 28 mai 2013.

La performance des étudiants a été mesurée via le rang de classement obtenu lors des ECN de 2013. Ces données ont été obtenues par confrontation de l'état civil des étudiants aux résultats publiés par le Centre national de gestion ${ }^{[10]}$. Cependant, comme le rang de classement aux ECN mesure principalement la performance des étudiants aux dossiers cliniques, qui comptent pour $90 \%$ du score global, nous avons recherché une mesure plus spécifique de la performance des étudiants pour la LCA. Nous avons ainsi également utilisé les scores (improprement appelés 
notes) d'examen de LCA obtenus par les étudiants en DCEM4 à Strasbourg, les scores des étudiants à Nancy n'étant pas disponibles.

Une analyse statistique descriptive de la participation a été réalisée dans un premier temps. Les caractéristiques des répondants ont été comparées à celles des non-répondants afin d'évaluer la présence d'un biais de sélection. Ces comparaisons ont été réalisées en utilisant le test du chi-deux ou le test de la somme des rangs de Wilcoxon (Mann-Whitney) en fonction du type de variable considéré. La distribution des valeurs des composantes de la motivation ne suivant pas tout à fait une loi normale, ces distributions ont été décrites en utilisant la médiane et l'intervalle interquartile. Les associations entre les différentes composantes de la motivation et, d'une part, les scores obtenus à l'examen de LCA de Strasbourg, et, d'autre part, le rang de classement aux ECN, ont été étudiées par l'estimation du coefficient de corrélation de Spearman. Le questionnaire en ligne a été administré au moyen du logiciel LimeSurvey et l'analyse statistique a été réalisée via le logiciel SAS version 9.2 (SAS Institute, Cary, North Carolina).

\section{Résultats}

Au total, 579 étudiants en DCEM4 ont été invités à participer à cette étude. Parmi eux, 205 (35,4\%) ont complété le questionnaire, dont respectivement 104 et 101 étudiants étaient inscrits dans les facultés de médecine de Strasbourg et de Nancy. Chez les participants, la proportion de femmes était de $60 \%$, l'âge médian de 25 ans (intervalle interquartile 24-25 ans) et le rang de classement médian aux ECN était 4013 (intervalle interquartile 2 564-5 970).

Les caractéristiques des non-répondants étaient similaires concernant la faculté de médecine d'origine (test du chi-deux $p=0,20$ ), le sexe (test du chideux $p=0,88$ ), et le rang de classement aux ECN (test de Wilcoxon $p=0,52$ ). L'âge était sensiblement plus élevé chez les non-participants (test de Wilcoxon $p=$ $0,01)$, les étudiants les plus âgés ayant un peu moins participé à cette étude (âge médian 25 ans, intervalle interquartile 24-26 ans).
Les résultats obtenus pour les différentes composantes de la motivation figurent dans le tableau I. La motivation extrinsèque était plus importante que la motivation intrinsèque, avec une médiane de scores de 5,00 versus 3,67. En ce qui concerne les autres composantes de la motivation, la perception d'autonomie et de contrôle était la plus forte (médiane de 4,67), suivie de la perception de la valeur de la tâche (médiane de 4,00). Le sentiment d'efficacité personnelle était la composante motivationnelle la plus altérée, avec un score médian de 3,33. Ces résultats étaient retrouvés de manière similaire à Strasbourg et à Nancy (résultats non détaillés).

Les corrélations entre ces différentes composantes et les résultats obtenus lors de l'examen de LCA par les étudiants de Strasbourg figurent dans le tableau II. Seul le SEP était corrélé de manière statistiquement significative, les étudiants étant d'autant plus performants que leur SEP était élevé (coefficient de corrélation de Spearman 0,242, $p=0,018)$. La motivation extrinsèque semblait également jouer positivement sur la performance des étudiants (coefficient de corrélation 0,118 ) sans toutefois atteindre la significativité statistique $(p=0,249)$.

Lorsque l'on s'intéresse à la performance mesurée par le rang de classement obtenu aux ECN (table 3), aucune des composantes de la motivation n'était plus associée à la performance de manière statistiquement significative. En particulier, le coefficient de corrélation de la perception d'efficacité avait une valeur très proche de zéro $(0,066, p=0,363)$. La motivation extrinsèque était la composante la plus associée au rang de classement, sans pour autant que cette association soit significative (coefficient de corrélation $0,129, p=0,077$ ).

\section{Discussion}

Cette étude montre que la composante motivationnelle la plus altérée chez les étudiants en médecine pour la pratique de la LCA est le sentiment d'efficacité personnelle. Ceci avait des conséquences en termes de performance, les étudiants avec une meilleure perception d'efficacité ayant obtenu de meilleurs scores à l'examen de LCA à Strasbourg. 
Tableau I. Composantes de la motivation des etudiants en DCEM4 pour l'apprentissage des competences en epidemiologie et biostatistique nécessaires à la LCA $(n=205)$.

\begin{tabular}{|l|c|c|}
\hline Composante & Médiane & $\begin{array}{c}\text { Intervalle } \\
\text { interquartile }\end{array}$ \\
\hline Motivation intrinsèque (MI) & 3,67 & {$[2,67-4,33]$} \\
\hline Motivation extrinsèque (ME) & 5,00 & {$[4,00-6,00]$} \\
\hline Perception de la valeur de la tâche (PVT) & 4,00 & {$[3,00-5,00]$} \\
\hline Perception de contrôle (PCO) & 4,67 & {$[4,00-5,33]$} \\
\hline Sentiment d'efficacité personnelle (SEP) & 3,33 & {$[2,67-4,33]$} \\
\hline
\end{tabular}

IC $95 \%$, Intervalle de confiance à $95 \%$.

LCA : Lecture critique d'article ; DCEM4 : quatrième année du deuxième cycle des études médicales.

Tableau II. Corrélations entre les composantes de la motivation et les scores obtenus à l'examen de LCA par les étudiants en DCEM4 de Strasbourg $\left(n=96^{*}\right)$.

\begin{tabular}{|l|c|c|c|c|c|c|}
\hline $\begin{array}{l}\text { Coefficients de corréla- } \\
\text { tion de Spearman }(\boldsymbol{p})\end{array}$ & $\begin{array}{c}\text { Score de } \\
\text { LCA }\end{array}$ & MI & ME & PVT & PCO & SEP \\
\hline Score de LCA & 1 & & & & & \\
\hline MI & $\begin{array}{c}-0,012 \\
(0,904)\end{array}$ & 1 & & & & \\
\hline ME & $\begin{array}{c}0,118 \\
(0,249)\end{array}$ & $\begin{array}{c}0,233 \\
(0,022)\end{array}$ & 1 & & & \\
\hline PVT & $\begin{array}{c}-0,024 \\
(0,816)\end{array}$ & $\begin{array}{c}0,565 \\
(<0,001)\end{array}$ & $\begin{array}{c}0,283 \\
(0,005)\end{array}$ & 1 & & \\
\hline PCO & $\begin{array}{c}-0,004 \\
(0,973)\end{array}$ & $\begin{array}{c}0,323 \\
(0,001)\end{array}$ & $\begin{array}{c}0,400 \\
(<0,001)\end{array}$ & $\begin{array}{c}0,293 \\
(0,004)\end{array}$ & 1 & $0,0,376$ \\
\hline SEP & $\begin{array}{c}0,242 \\
(0,018)\end{array}$ & $\begin{array}{c}0,393 \\
(<0,001)\end{array}$ & $\begin{array}{c}0,360 \\
(<0,001)\end{array}$ & $\begin{array}{c}0,248 \\
(0,015)\end{array}$ & $(<0,001)$ & 1 \\
\hline
\end{tabular}

* Le score obtenu à l'examen de LCA n'était pas disponible pour 8 étudiants à Strasbourg.

LCA : Lecture critique d'article; DCEM4: Quatrième année du deuxième cycle des études médicales; MI, Motivation intrinsèque; ME: Motivation extrinsèque; PVT: Perception de la valeur de la tâche; PCO : Perception de contrôle ; SEP : Sentiment d'efficacité personnelle.

D'une manière attendue, nous avons trouvé que le niveau de motivation extrinsèque était supérieur à celui de la motivation intrinsèque. Ceci s'explique volontiers par le contexte des ECN, dans la mesure où les résultats de ces épreuves conditionnent le choix de la spécialité et du lieu d'exercice des futurs praticiens. Ceci constitue une source de motivation extrinsèque importante. Plus étonnamment, la $\mathrm{ME}$ semblait davantage associée à la performance que la MI. Ceci pourrait sembler aller à l'encontre de la théorie de l'autodétermination, où un niveau de motivation intrinsèque élevé est théoriquement associé à de meilleures performances en termes d'apprentissage ${ }^{[6]}$. Il est toutefois utile de souligner qu'il existe, telles que décrites dans la littérature, différentes sources de régulation de la ME (externe, introjectée et identifiée) correspondant à des niveaux croissants d'autodétermination ${ }^{[6]}$ :

- La régulation externe, qui correspond à la définition communément admise de la ME (recherche de récompenses et évitement de sanctions).

- La régulation introjectée, qui provoque des comportements dictés par la représentation que se fait l'individu de leurs conséquences dans un registre 
Tableau III. Correlations entre les composantes de la motivation et le rang de classement obtenu aux ECN par les etudiants en DCEM4 de Strasbourg et de Nancy $\left(n=190^{*}\right)$.

\begin{tabular}{|l|c|c|c|c|c|c|}
\hline $\begin{array}{l}\text { Coefficients de corréla- } \\
\text { tion de Spearman }(\boldsymbol{p})\end{array}$ & $\begin{array}{c}\text { Rang } \\
\text { ECN** }\end{array}$ & MI & ME & PVT & PCO & SEP \\
\hline Rang ECN** & 1 & & & & & \\
\hline MI & $\begin{array}{c}-0,098 \\
(0,179)\end{array}$ & 1 & & & & \\
\hline ME & $\begin{array}{c}0,129 \\
(0,077)\end{array}$ & $\begin{array}{c}0,161 \\
(0,027)\end{array}$ & 1 & & & \\
\hline PVT & $\begin{array}{c}0,061 \\
(0,403)\end{array}$ & $\begin{array}{c}0,553 \\
(<0,001)\end{array}$ & $\begin{array}{c}0,311 \\
(<0,001)\end{array}$ & 1 & & \\
\hline PCO & $\begin{array}{c}-0,071 \\
(0,329)\end{array}$ & $\begin{array}{c}0,285 \\
(<0,001)\end{array}$ & $\begin{array}{c}0,194 \\
(0,073)\end{array}$ & $\begin{array}{c}0,277 \\
(0,001)\end{array}$ & 1 & \\
\hline SEP & $\begin{array}{c}0,066 \\
(0,363)\end{array}$ & $\begin{array}{c}0,340 \\
(<0,001)\end{array}$ & $\begin{array}{c}0,288 \\
(<0,001)\end{array}$ & $\begin{array}{c}0,303 \\
(<0,001)\end{array}$ & $\begin{array}{c}0,284 \\
(<0,001)\end{array}$ & 1 \\
\hline
\end{tabular}

* Le classement aux ECN n'était pas disponible pour 15 étudiants.

** L'opposé du rang de classement a été utilisé : les valeurs des coefficients de corrélation supérieurs à 0 indiquent une performance plus élevée.

ECN : Épreuves classantes nationales ; DCEM4 : Quatrième année du deuxième cycle des études médicales ; Rang ECN : Rang de classement aux ECN; MI : Motivation intrinsèque; ME : Motivation extrinsèque ; PVT : Perception de la valeur de la tâche ; PCO : Perception de contrôle ; SEP : Sentiment d'efficacité personnelle.

psychoaffectif (par exemple, privilégier l'internat de chirurgie par crainte d'être mal vu par son entourage familial, constitué de chirurgiens).

- La régulation identifiée, qui se caractérise par la reconnaissance d'une valeur instrumentale aux comportements adoptés : le sujet va participer à une activité parce qu'elle est importante pour lui, même si elle n'est pas nécessairement intéressante. La formation devient alors l'instrument de la réalisation des projets personnels ou professionnels de l'individu.

Ainsi, les ECN - passage incontournable en vue du choix des modalités d'exercice des futurs médecins - pourraient être à l'origine d'un fort niveau de ME par régulation identifiée. Celle-ci s'accompagnerait d'un niveau d'autodétermination élevé, ce qui pourrait expliquer la meilleure performance en LCA des étudiants ayant un niveau de ME élevé.

Concernant les autres composantes motivationnelles, nous avons identifié que la PCO était élevée. Ce résultat est particulièrement cohérent avec le contrôle important dont disposent les étudiants quant aux stratégies de gestion de l'environnement d'apprentissage de la LCA : choix d'assister aux cours, de suivre des conférences, de participer à des ECN blanches, etc.

La PVT se situait quant à elle à un niveau intermédiaire, ce qui semble traduire la bonne perception par les étudiants de l'intérêt de disposer des connaissances méthodologiques pour pouvoir pratiquer la LCA en vue de leur exercice futur. Les stratégies d'enseignement qui ont probablement contribué à ce résultat comportent notamment l'énoncé des buts d'apprentissage en termes d'objectifs éducationnels et de compétences visées ${ }^{[6]}$.

Finalement, le SEP était la composante motivationnelle la plus altérée. Or, les étudiants ont plutôt tendance à s'engager dans des tâches pour lesquelles ils se sentent compétents et, à l'inverse, à éviter celles pour lesquelles ils ne se sentent pas compétents $^{[9,11,12]}$. En effet, plus un apprenant a un SEP élevé, plus il choisit des activités qui suscitent un défi et lui donnent l'occasion d'entreprendre des apprentissages complexes ${ }^{[13]}$. Par ailleurs, il aura tendance à se fixer des objectifs élevés, à réguler ses efforts, à persévérer en situation d'échec, et à mieux gérer son stress et son anxiété, ce qui génère par conséquent de meilleures performances. 
Les résultats de notre étude mettent ainsi en évidence que le principal enjeu pédagogique lié à l'apprentissage de la LCA consiste à définir des stratégies visant à renforcer le SEP. Selon Bandura, la perception qu'une personne a de sa compétence est déterminée par quatre sources principales : ses expériences ou ses performances antérieures, ses observations d'autres personnes, la persuasion verbale et ses états psychologiques et émotifs ${ }^{[14]}$. Le SEP n'est ainsi pas le reflet des capacités réelles de l'individu, mais la croyance qu'il a en ses capacités. De ce fait, plusieurs grands principes d'interventions pourraient être adaptés au cadre de l'enseignement de la $\mathrm{LCA}^{[15]}$ :

- Fournir des outils et des stratégies qui permettraient aux étudiants de s'auto-évaluer le plus justement possible. En effet, les étudiants ont parfois des difficultés à avoir une perception réaliste de leurs capacités à accomplir les tâches académiques demandées. Il est ainsi primordial qu'ils n'aient pas une vision erronée, et en particulier sous-estimée, de leur véritable compétence pour la LCA. Au-delà de la proposition d'épreuves de LCA à un niveau local (ECN blanches, conférences), la mise en place de la plateforme SIDES pourrait prochainement fournir, au niveau national, des outils d'autoévaluation aux étudiants, en mettant à leur disposition des sujets corrigés issus des différentes facultés de médecine françaises ${ }^{[16]}$.

- Les enseignants de LCA ont également un rôle capital à jouer au moyen des réflexions qu'ils adressent aux étudiants, des réponses apportées aux questions posées et des rétroactions offertes aux étudiants en matière de performance. La rétroaction sur les performances obtenues sur la seule base d'une note étant préjudiciable pour les étudiants en situation d'échec, il serait très utile de proposer un bref entretien à ces étudiants, visant notamment à définir avec eux des objectifs d'apprentissage leur permettant de progresser, et à souligner les aspects positifs de leur travail. Il convient cependant de noter que la persuasion verbale de la part de l'enseignement pour renforcer le SEP peut s'avérer plus ou moins efficace selon les étudiants.
- Enseigner aux élèves à devenir compétents peut être plus profitable que d'essayer de les persuader qu'ils le sont. Il s'agit donc, pour l'enseignant, de montrer aux étudiants comment utiliser efficacement les différentes stratégies d'apprentissage, en particulier métacognitives, dans l'optique d'améliorer leur performance ${ }^{[6]}$.

Ce travail de recherche présente plusieurs limites. D'abord, la participation était de 35,4\%, ce qui pourrait laisser entendre que seuls les étudiants les plus motivés ont répondu au questionnaire. Cependant, bien que les étudiants les plus âgés aient sensiblement moins participé à cette étude, les caractéristiques des répondants étaient semblables à celles des non-répondants en ce qui concerne la faculté d'origine, le sexe et le rang de classement aux ECN, ce qui rend peu vraisemblable l'existence d'un biais de sélection.

Une autre limite de ce travail concerne le risque d'altération des propriétés psychométriques du MSLQ suite à la traduction en français et à la réduction du nombre d'items de 26 à 15 . En effet, un travail préalable d'adaptation transculturelle assorti d'une étude de la validité du nouvel outil de mesure aurait pu être envisagé ${ }^{[17]}$. Cependant, bien que l'étape de rétro-traduction en anglais n'ait pas été réalisée ici, la version traduite du MSLQ a été testée auprès de représentants étudiants afin de vérifier la bonne compréhensibilité des différents items. De plus, les questions retenues correspondaient aux trois questions qui exploraient le mieux les différentes composantes étudiées. Enfin, les valeurs des coefficients de corrélation entre les dimensions retrouvées dans notre étude sont proches de ceux de l'étude de validation du MSLQ ${ }^{[8]}$, ce qui laisse présumer une préservation acceptable des propriété psychométriques de l'outil utilisé.

Ensuite, comme l'examen de LCA à Strasbourg s'est déroulé avant l'administration du questionnaire, un lien de causalité inverse entre le score obtenu lors de l'examen de LCA en DCEM4 et le niveau de SEP ne peut pas être exclu. Compte tenu de l'importance du feed-back sur cette composante de la motivation, il est effectivement possible que le score de LCA ait affecté le niveau de SEP. Cependant, il est très 
probable que le niveau de SEP ait été déterminé principalement par d'autres facteurs antérieurs à cet examen et itératifs, comme les notes obtenues lors des années antérieures, les ECN blanches, l'observation d'autres personnes et les échanges réalisés avec les enseignants lors des diverses séances d'enseignement de la LCA.

Enfin, l'utilisation du rang de classement global aux ECN est un critère de jugement qui manque de spécificité pour mesurer la performance des étudiants pour la LCA, puisque l'épreuve de LCA ne compte que pour $10 \%$ du score global aux ECN. Une perspective intéressante de ce travail pourrait ainsi consister en l'analyse des scores de LCA obtenus par ces étudiants aux ECN, ce qui constituerait un critère spécifique dans l'optique d'étudier la performance des étudiants pour la LCA.

\section{Contributions}

Jérémie Jégu et Thierry Pelaccia ont défini le sujet de recherche et les modalités de déroulement de l'étude. Marc Braun et Jérémie Jégu ont participé à la collecte des données. Jérémie Jégu a rédigé la première version du manuscrit. Thierry Pelacia et Marc Braun ont contribué à la rédaction en réalisant une lecture critique du manuscrit.

\section{Remerciements}

Nous remercions les services administratifs des facultés de médecine de Strasbourg et de Nancy, ainsi que les délégués étudiants pour leur précieuse contribution à la mise en place de cette étude.

\section{Déclarations d'intérêt}

Les auteurs de ce manuscrit n'ont aucun conflit d'intérêt à déclarer.

\section{Approbation éthique}

Ce projet de recherche a obtenu un avis favorable de la part du Comite d'éthique des facultés de médecine, d'odontologie, de pharmacie et du CHR de Strasbourg (dossier $\mathrm{N}^{\circ}$ AMK/BG/2013/C5), et a fait l'objet d'une déclaration normale auprès de la Commission nationale informatique et libertés -CNIL(déclaration $\mathrm{N}^{\circ} 1669705 \mathrm{v}$ 0).

\section{Valorisation scientifique}

Ce travail de recherche a fait l'objet d'une communication orale lors des $2^{\text {èmes }}$ États généraux de la formation et de la recherche médicales à Bobigny (5 et 6 décembre 2013).

\section{Références}

1. Windish DM, Huot SJ, Green ML. Medicine residents' understanding of the biostatistics and results in the medical literature. JAMA 2007;298:1010-22.

2. Czernichow P, Fillastre J-P, Lavoinne A, Lemeland J-F, Roussel $\mathrm{F}$. Facteurs liés à la concordance des corrections d'une épreuve de lecture critique d'article. Pédagogie Médicale 2006;7:82-90.

3. Lorette G, Jolly D, Ambrosi P, Dreyfuss D, Magne J-L, Kohler C, Duhaut P, Gérard J-L. Influence de la lecture critique d'articles sur les classements des étudiants aux épreuves classantes nationales 2009. Presse Med 2010;39:e118-25.

4. Jolly D, Lorette G, Ambrosi P, Dreyfuss D, Magne J-L, Kohler C, Duhaut P, Gérard J-L, Rogez J-M. Les épreuves classantes nationales (ECN) 2010 : résultats, classements, influence de la lecture critique d'articles. Presse Med 2011;40:447-8.

5. Jolly D, Lorette G, Ambrosi P, Dreyfuss D, Chaffanjon P, Kohler C, Duhaut P, Rogez J-M. Résultats des épreuves classantes nationales (ECN) 2011. Presse Med 2012;41:323-5.

6. Pelaccia T, Delplancq H, Triby E, Leman C, Bartier J-C, Dupeyron J-P. La motivation en formation : une dimension réhabilitée dans un environnement 
d'apprentissage en mutation. Pédagogie Médicale 2008;9:103-21.

7. Ryan, Deci. Intrinsic and Extrinsic Motivations: Classic Definitions and New Directions. Contemp Educ Psychol 2000;25:54-67.

8. Pintrich PR, Smith DAF, Garcia T, Mckeachie WJ. Reliability and Predictive Validity of the Motivated Strategies for Learning Questionnaire (MSLQ). Educational and Psychological Measurement 1993;53: 801-13.

9. Thierry Pelaccia HD. Impact of training periods in the emergency department on the motivation of health care students to learn. Med Educ 2009;43:462-9.

10. Liste des étudiants et des internes de médecine, classés par ordre de mérite, ayant satisfait aux épreuves classantes nationales anonymes donnant accès au troisième cycle des études médicales, organisées au titre de l'année universitaire 2013-2014. 2013 [On-line] Disponible sur : http://www.cng.sante.fr/IMG/ pdf/Liste_des_candiats_ECN_0507-2.pdf

11. Bandura A. Self-efficacy: The exercise of control. New York :Worth Publishers, 1997.
12. Pierce JL, Kostova T, Dirks KT. Toward a Theory of Psychological Ownership in Organizations. Acad Manage Rev 2001;26:298-310.

13. Galand B, Vanlede M. Le sentiment d'efficacité personnelle dans l'apprentissage et la formation : quel rôle joue-t-il ? D'où vient-il ? Comment intervenir ? Savoirs 2004;Hors série:91-116.

14. Rondier M. A. Bandura. Auto-efficacité. Le sentiment d'efficacité personnelle. L'orientation scolaire et professionnelle 2004;33:475-6.

15. Viau R. La motivation à apprendre en milieu scolaire. Québec: ERPI, 2009.

16. Présentation des projets ECNi et SIDE-S [Internet]. Site internet du Système Informatique Distribué d'Evaluation en Santé, 2013.

17. Beaton DE, Bombardier C, Guillemin F, Ferraz MB. Guidelines for the process of cross-cultural adaptation of self-report measures. Spine 2000;25:3186-91.

Correspondance et offprints : Jérémie Jégu, Laboratoire d'épidémiologie et de santé publique, Faculté de médecine, Université de Strasbourg, 4 rue Kirschleger, 67000 Strasbourg, France.

Téléphone : +33 (0)3 68854026 / Télécopie : +33 (0)3 68853189 . Mailto : jeremie.jegu@unistra.fr 